\title{
Bipolarity and the relational division
}

\author{
Nouredine Tamani ${ }^{1}$ Ludovic Liétard ${ }^{2}$ Daniel Rocacher ${ }^{3}$ \\ ${ }^{1}$ IRISA/ENSSAT/Univ. Rennes 1, tamani@enssat.fr \\ ${ }^{2}$ IRISA/IUT/Univ. Rennes 1, ludovic.lietard@univ-rennes1.fr \\ ${ }^{3}$ IRISA/ENSSAT/Univ. Rennes 1, rocacher@enssat.fr
}

\begin{abstract}
A fuzzy bipolar relation is a relation defined by a fuzzy bipolar condition, which could be interpreted as an association of a constraint and a wish. In this context, the extension of the relational division operation to bipolarity is studied in this paper. Firstly, we define a bipolar division when the involved relations are crisp. Then, we define, from the semantic point of view, several forms of bipolar division when the involved relations are defined by fuzzy bipolar conditions. These forms of division can be related to the general model of fuzzy bipolar division introduced in $[4,5]$.
\end{abstract}

Keywords: Relational division, fuzzy bipolar conditions, bipolar division, tolerant bipolar division.

\section{Introduction}

The bipolarity in the domain of flexible querying is defined as the ability to express, in one single condition, positive and negative conditions, which are associated to form bipolar conditions. Several interpretations have been introduced for the combination and the evaluation of bipolar conditions (see $[1,8,9,12,13,14])$. In this paper, we rely on the interpretation introduced by Dubois and Prade [8, 9], in which a bipolar condition is made of a constraint $c$, which is mandatory, and a wish $w$, which is optional. It is noted $(c, w)$ and means "satisfy $c$ and if possible satisfy $w^{\prime \prime}$. We define then a bipolar query as a query that involves bipolar conditions. This paper is set in the general case of fuzzy bipolar conditions where $c$ and $w$ are fuzzy.

When querying a relation $R$ with a fuzzy bipolar condition, each tuple $t$ from $R$ is then attached with a pair of grades $\left(\mu_{c}(t), \mu_{w}(t)\right)$ that expresses the degree of its satisfaction respectively to the constraint $c$ and the wish $w$, and a so-called fuzzy bipolar relation is obtained.

In order to define a bipolar relational algebra, the algebraic operators (selection, projection, join, union, intersection) have been extended to fuzzy bipolar conditions $[11,6]$. These operators allow the expression of fuzzy bipolar queries over fuzzy bipolar relations.

In this paper, we are interested at the first step to the definition of a tolerant bipolar division operator, applied on boolean relations $R$ and $S$, where the underlying universal quantifier all of the division oper- ator is substituted by the linguistic quantifier almost all as in the following query "Find students who got a mark which is $\geq 10$ and if possible which is $\geq 15$ in almost all courses", which corresponds to a tolerant bipolar division query of the bipolar relation StudentResults $(\geq 10, \geq 15)$ by the boolean relation Courses. The interpretation of such a tolerant bipolar division is based on quantified propositions of type "Almost_all $S$ are $R$ ".

At the second step, we study several approaches to express division queries which involve fuzzy bipolar relations, such as the query "Find students who are well scored and if possible very well scored in all difficult and if possible in all very difficult courses", which corresponds to a bipolar division of the fuzzy bipolar relation StudentResults $_{(\text {Well, VeryWell) }}$ by the fuzzy bipolar relation Courses (Difficult, VeryDifficult) $_{\text {. }}$

The remainder of the paper is structured as follows. In section 2, a short reminder about fuzzy bipolar conditions and relations is introduced. In section 3, we recall previous works, introduced in $[4,5]$, to define a division operator applied on fuzzy bipolar relations. However, one may notice that this kind of bipolar division has not the form of an "and if possible" statement. In section 4, a tolerant division operator and its extension to bipolarity are proposed. In section 5, we introduce an interpretation of the division involving fuzzy bipolar relations, which is based on a final user's expectations. It is shown that this interpretation fits within the general model $[4,5]$. Finally, section 6 concludes the paper and draws some lines for future works.

\section{Fuzzy bipolar conditions}

A bipolar condition is a compound condition which is made of two conditions defined on the same universe: i) a constraint $c$, which describes the set of acceptable elements, and ii) a wish $w$ which defines the set of desired or wished elements. The negation of $c$ is the set of rejected elements since it describes non-acceptable elements. Since it is not coherent to wish a rejected element, the following property of coherence holds: $w \subseteq c$.

In addition, condition $c$ is mandatory since an element which does not satisfy $c$ is rejected $(\neg c$ is the negative pole of the bipolarity). Condition $w$ is optional since an element which does not satisfy it is not necessarily rejected ( $w$ is the positive pole of 
the bipolarity).

If $c$ and $w$ are boolean conditions, the satisfaction with respect to $(c, w)$ is a pair from $\{0,1\}^{2}$. When querying a database with such a condition, tuples satisfying the constraint and the wish are returned in priority to the user. If such answers do not exist, tuples satisfying only the constraint are delivered.

If $c$ and $w$ are fuzzy conditions (defined on the universe $U$ ), the property of coherence becomes: $\forall u \in U, \mu_{w}(u) \leq \mu_{c}(u)$ and the satisfaction with respect to $(c, w)$ is a pair of degrees from $[0,1] \times[0,1]$. Each element $u$ from $U$ is then attached with a pair of grades $\left(\mu_{c}(u), \mu_{w}(u)\right)$ that expresses the degree of its satisfaction respectively to the constraint $c$ and to the wish $w$. In the context of bipolar relations, a tuple $t$ is then denoted $\left(\mu_{c}, \mu_{w}\right) / t$. We assume that any tuple $u$ such that $\mu_{c}(u)=0$ does not belong to the fuzzy bipolar relation.

In order to rank the delivered tuples, previous bipolar approaches $[8,9,12,13,14]$ consider the aggregation of the constraint and the wish. In our case, the constraint and the wish are not commensurable and tuples cannot be ranked from the most preferred to the least preferred using an aggregation of $\mu_{c}$ and $\mu_{w}$. However they can be ranked using the lexicographical order: $t_{1}$ is preferred to $t_{2}$, denoted $t_{1}>t_{2}$ or $\left(\mu_{c}\left(t_{1}\right), \mu_{w}\left(t_{1}\right)\right)>\left(\mu_{c}\left(t_{2}\right), \mu_{w}\left(t_{2}\right)\right)$, if and only if $\mu_{c}\left(t_{1}\right)>\mu_{c}\left(t_{2}\right)$ or

$$
\left(\mu_{c}\left(t_{1}\right)=\mu_{c}\left(t_{2}\right) \wedge \mu_{w}\left(t_{1}\right)>\mu_{w}\left(t_{2}\right)\right) .
$$

These two degrees being not commensurable and since the constraint is mandatory, its satisfaction is firstly used to discriminate among answers. The satisfaction with respect to the wish being not mandatory, it can only be used to discriminate among answers having the same evaluation with respect to the constraint. A total order is then obtained on $c$ and $w$ (with $(1,1)$ as the greatest element and $(0,0)$ as the least element).

Based on the lexicographical order, the $\operatorname{lmin}$ and lmax operators $[10,6]$ are introduced in order to define the conjunction (resp. intersection) and the disjunction (resp. union) of bipolar conditions (resp. relations). They are respectively defined as follows:

$$
\begin{aligned}
& ([0,1] \times[0,1])^{2} \rightarrow[0,1] \times[0,1] \\
& \left((\mu, \eta),\left(\mu^{\prime}, \eta^{\prime}\right)\right) \mapsto \operatorname{lmin}\left((\mu, \eta),\left(\mu^{\prime}, \eta^{\prime}\right)\right)= \\
& \begin{cases}(\mu, \eta) & \text { if } \mu<\mu^{\prime} \vee\left(\mu=\mu^{\prime} \wedge \eta<\eta^{\prime}\right), \\
\left(\mu^{\prime}, \eta^{\prime}\right) & \text { else. }\end{cases} \\
& ([0,1] \times[0,1])^{2} \rightarrow[0,1] \times[0,1] \\
& \left((\mu, \eta),\left(\mu^{\prime}, \eta^{\prime}\right)\right) \mapsto \operatorname{lmax}\left((\mu, \eta),\left(\mu^{\prime}, \eta^{\prime}\right)\right)= \\
& \begin{cases}(\mu, \eta) & \text { if } \mu>\mu^{\prime} \vee\left(\mu=\mu^{\prime} \wedge \eta>\eta^{\prime}\right), \\
\left(\mu^{\prime}, \eta^{\prime}\right) & \text { else. }\end{cases}
\end{aligned}
$$

The $\operatorname{lmin}$ (resp. lmax) operator is commutative, associative, idempotent and monotonic. The pair of grades $(1,1)$ is the neutral (resp. absorbing) element of the operator $\operatorname{lmin}$ (resp. lmax) and the pair $(0,0)$ is the absorbing (resp. neutral) element of the operator lmin (resp. lmax).

It can be noticed that a fuzzy relation is a fuzzy bipolar relation such that $\forall x,\left(\mu_{c}(x)=\mu_{w}(x)\right)$, which means that a fuzzy condition is a bipolar condition in which the wish is equal to the constraint; and a regular relation corresponds to a fuzzy bipolar relation in which the pair of grades $(1,1)$ is associated to their tuples. It has also been proven that lmin (resp. lmax) generalizes the triangular norm min (resp. the triangular co-norm $\max$ ) [10, 6].

\section{Fuzzy and bipolar division operations}

Two lines of extension of the relational division have been proposed by Bosc et al. in [7]. The first one concerns the operands of the division operator and the second one is about the operator itself. In this section, we are interested in the extension of the operands to fuzzy bipolar relations.

The definition of the division operator involving fuzzy bipolar relations is based on the extension of the division operator to fuzzy relations (obtained from fuzzy queries). In subsection 3.1, a short reminder of the division involving fuzzy relations is introduced and in subsection 3.2, we describe the general model $[4,5]$ for the extension of the relational division operator to fuzzy bipolar relations.

\subsection{The division of fuzzy relations}

We describe in what follows, the extension of the division operator to fuzzy relations proposed in [7].

Let $R$ and $S$ be two fuzzy relations of schema $(A, X)$ and $B$ respectively, where $A$ and $B$ are compatible sets of attributes.

The relational division of $R(A, X)$ by $S(B)$, denoted $R[A \div B] S$ or more simply $R \div S$, defines a relation which consists of elements from $R[X]$ which are associated to every element of $S$, where $R[X]$ is the projection of $R$ on attributes $X$. The grades attached to the resulting tuples are defined as follows:

$$
\mu_{R \div S}(x)=i n f_{a \in S} \mu_{S}(a) \rightarrow \mu_{R}(x, a),
$$

where $\rightarrow$ is a fuzzy implication. We recall that any fuzzy implication operator satisfies the following properties (which are used later):

$$
\begin{aligned}
& \text { - } x \leq z \Rightarrow(x \rightarrow y) \geq(z \rightarrow y) \\
& \text { - } y \leq z \Rightarrow(x \rightarrow y) \leq(x \rightarrow z) \\
& \text { - } \forall x,(x \rightarrow 1)=1 \\
& \text { - } \forall y,(0 \rightarrow y)=1 \\
& \text { - }(1 \rightarrow 0)=0
\end{aligned}
$$

Depending on the choice of the fuzzy implication, several meanings can be obtained for $\mu_{R \div S}(x)$. For instance, when a Gödel fuzzy implication $\left(a \rightarrow_{G \ddot{o}}\right.$ $b=1$ if $a \leq b ; b$ else) is used, the degree $\mu_{S}$ is viewed as a threshold to be reached by $\mu_{R}$. In this case, $\mu_{R \div S}(x)=1$ if all thresholds are reached, otherwise, $\mu_{R \div S}(x)$ corresponds to the smallest value $\mu_{R}$ that does not reach its threshold. 


\begin{tabular}{|c|c|c|}
\hline \#Student & \#Course & $\mu_{\text {Well Scored }}$ \\
\hline \hline 1 & $C_{1}$ & 1 \\
\hline 1 & $C_{2}$ & 0.6 \\
\hline 1 & $C_{3}$ & 0.9 \\
\hline 2 & $C_{1}$ & 0.9 \\
\hline 2 & $C_{2}$ & 0.6 \\
\hline 2 & $C_{3}$ & 0.8 \\
\hline 3 & $C_{1}$ & 0.4 \\
\hline 3 & $C_{3}$ & 0.8 \\
\hline
\end{tabular}

Table 1: Example of extension of the relation $R_{W e l l}$.

\begin{tabular}{|c|c|}
\hline \#Course & $\mu_{\text {Difficult }}$ \\
\hline \hline$C_{1}$ & 1 \\
\hline$C_{2}$ & 0.5 \\
\hline$C_{3}$ & 0.8 \\
\hline
\end{tabular}

Table 2: Example of extension of the relation $S_{D i f}$.

Example 1: The following query "Find students who are well scored in every difficult courses" corresponds to the division of the fuzzy relation StudentResults ${ }_{W e l l}$, denoted $R_{W e l l}$, by the fuzzy relation Courses ${ }_{\text {Difficult }}$, denoted $S_{\text {Dif }}$.

Tables 1, 2 and 3 display respectively examples of relations StudentResults Well $_{\text {, Courses }}$ Difficult and the fuzzy relation which results from the division, when Gödel fuzzy implication is used. The resulting relation states that for the student \#1 all thresholds are reached, and for the student \#2, thresholds are reached till 0.9 .

\subsection{The division of bipolar relations}

A general model for the division of bipolar relations is introduced in $[4,5]$. It is based on the decomposition of the division operation involving fuzzy bipolar relations on two divisions involving fuzzy relations. The two obtained relations are, after that, combined to form the resulting fuzzy bipolar relation.

Let $R_{(c, w)}(A, X)$ and $S_{\left(c^{\prime}, w^{\prime}\right)}(B)$ be two fuzzy bipolar relations defined respectively by fuzzy bipolar conditions $(c, w)$ and $\left(c^{\prime}, w^{\prime}\right)$. The fuzzy relations which can be extracted from them are:

- $R_{c}$ and $R_{w}$ of schema $(A, X)$ which are respectively the projection of $R_{(c, w)}$ on the constraint $c$ and the wish $w$.

- $S_{c^{\prime}}$ and $S_{w^{\prime}}$ of schema $B$ which are respectively the projection of $S_{\left(c^{\prime}, w^{\prime}\right)}$ on the constraint $c^{\prime}$ and the wish $w^{\prime}$.

The fuzzy division operations which can be performed are (where $S$ is the set made of $B$-values

\begin{tabular}{|c|c|}
\hline \#Student & $\mu_{R_{\text {Well }} \div S_{\text {Dif }}}$ \\
\hline \hline 1 & 1 \\
\hline 2 & 0.9 \\
\hline
\end{tabular}

Table 3: The resulting relation from $R_{W e l l} \div S_{D i f}$. appearing in $S_{\left(c^{\prime}, w^{\prime}\right)}$, and $\rightarrow$ is a fuzzy implication):

$R_{c} \div S_{w^{\prime}}$ : the degrees of satisfaction of the resulting tuples are obtained by the formula (1):

$$
\mu_{R_{c} \div S_{w^{\prime}}}(x)=i n f_{a \in S}\left(\mu_{w^{\prime}}(a) \rightarrow \mu_{c}(x, a)\right)
$$

$R_{w} \div S_{c^{\prime}}$ : the degrees of satisfaction of the resulting tuples are obtained by the formula (2):

$$
\mu_{R_{w} \div S_{c^{\prime}}}(x)=i n f_{a \in S}\left(\mu_{c^{\prime}}(a) \rightarrow \mu_{w}(x, a)\right)
$$

$R_{c} \div S_{c^{\prime}}$ : the degrees of satisfaction of the resulting tuples are obtained by the formula (3):

$$
\mu_{R_{c} \div S_{c^{\prime}}}(x)=i n f_{a \in S}\left(\mu_{c^{\prime}}(a) \rightarrow \mu_{c}(x, a)\right)
$$

$R_{w} \div S_{w^{\prime}}$ : the degrees of satisfaction of the resulting tuples are obtained by the formula (4):

$$
\mu_{R_{w} \div S_{w^{\prime}}}(x)=i n f_{a \in S}\left(\mu_{w^{\prime}}(a) \rightarrow \mu_{w}(x, a)\right)
$$

The pair of grades of satisfaction proposed in this approach is the one composed by the extremum values $\mu_{R_{c}} \div S_{w^{\prime}}$ (formula (1)) and $\mu_{R_{w} \div S_{c^{\prime}}}$ (formula (2)), since they are the two degrees which respect the coherence property of bipolar conditions (due to the properties of fuzzy implications which ensure that $\forall x, \mu_{R_{w}} \div S_{c^{\prime}}(x) \leq \mu_{R_{c} \div S_{c^{\prime}}}(x) \leq \mu_{R_{c} \div S_{w^{\prime}}}(x)$ and $\left.\forall x, \mu_{R_{w} \div S_{c^{\prime}}}(x) \leq \mu_{R_{w} \div S_{w^{\prime}}}(x) \leq \mu_{R_{c} \div S_{w^{\prime}}}(x)\right)$, and guarantee that the resulting fuzzy bipolar relation is a quotient $[4,5]$.

The degree $\mu_{R_{c}} \div S_{w^{\prime}}$ expresses at what extent a perfect element in the divisor $S$ is related to an accepted element in the dividend $R$, and the degree $\mu_{R_{w}} \div S_{c^{\prime}}$ expresses at what extent an accepted element of $S$ is related to a preferred element of $R$.

The degrees attached to each answer are established as the only possible alternative, without taking into account the bipolar aspect of the resulting relation. In other terms, this division does not take the form of an "and if possible" statement, and we think that it is a major issue. So, we propose other interpretations for the division extended to bipolarity in the next sections. Firstly, a tolerant bipolar division applied on boolean relations is studied in section 4; secondly, several forms of division involving fuzzy bipolar relations is discussed in section 5 .

\section{Tolerant bipolar division of boolean relations}

This section is devoted to a tolerant division in which the underlying quantifier is the linguistic quantifier almost all. Subsection 4.1 introduces the basis of such a division. An improvement based on bipolarity is proposed in subsection 4.2.

\subsection{A tolerant relational division}

Let $R(A, X)$ and $S(B)$ be two regular (boolean) relations with $(A, X)$ and $B$ are their respective schemas. A tolerant division of $R$ by $S$, denoted 


\begin{tabular}{|c|c||c|c|}
\hline \#Student & \#Course & \#Student & \#Course \\
\hline \hline 1 & $C_{1}$ & 2 & $C_{1}$ \\
\hline 1 & $C_{2}$ & 2 & $C_{2}$ \\
\hline 1 & $C_{3}$ & 2 & $C_{3}$ \\
\hline 1 & $C_{4}$ & 2 & $C_{4}$ \\
\hline 1 & $C_{5}$ & 2 & $C_{5}$ \\
\hline 3 & $C_{1}$ & 4 & $C_{1}$ \\
\hline 3 & $C_{2}$ & 4 & $C_{3}$ \\
\hline 3 & $C_{3}$ & 4 & $C_{4}$ \\
\hline 3 & $C_{5}$ & 4 & $C_{5}$ \\
\hline
\end{tabular}

Table 4: Extension of the relation StudentResults $\geq 10$.

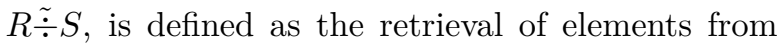
$R[X]$ which are related to almost all elements of $S$.

The interpretation of such a division is based on that of quantified propositions of the form " $Q Y$ are $Z$ ". More precisely, $R \stackrel{\sim}{\div} S$ delivers elements $x$ from $R[X]$ where $x$ is attached to the degree of satisfaction of the quantified proposition: "Almost all elements of $S$ are related to $x$ in $R$ ". Therefore, for a given value $x$ appearing in $R$ we get:

$$
\mu_{R[A \tilde{\div} B] S}(x)=\mu_{\text {Almost_all }}\left(\frac{v}{n}\right)
$$

where $v$ is the number of elements of $S$ which are in relationship $R$ with $x$ and $n$ is the cardinality of $S$.

This tolerant division delivers elements which would be discarded by the regular division $R \div S$.

Example 2: Let Courses(\#Course) and StudentResults(\#Student, \#Course, Mark) be two crisp relations. The following query "Find students who got a mark which is $\geq 10$ in almost all courses" corresponds to the tolerant division

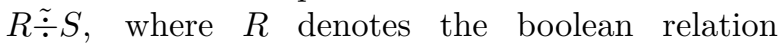
StudentResults $s_{\geq 10}$, which is a projection on columns \#Student and \#Course of the restriction of the relation StudentResults to elements which got a mark $\geq 10$, and $S$ is the relation Courses.

Let table 4 be an example of the relation StudentResults $s_{\geq 10}$ and Courses is a table which contains 5 courses: $C_{1}, C_{2}, C_{3}, C_{4}$ and $C_{5}$.

We define the linguistic quantifier almost all by the following membership function:

$$
\begin{aligned}
\mu_{\text {Almost_all }}:[0,1] & \rightarrow[0,1] \\
p & \mapsto \mu_{\text {Almost_all }}(p)= \begin{cases}0 & \text { if } p \in[0,0.5] \\
2 p-1 & \text { if } p \in[0.5,1]\end{cases}
\end{aligned}
$$

The resulting relation from this tolerant division is a fuzzy relation (table 5 ), in which:

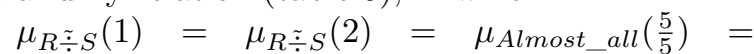
$\mu_{\text {Almost_all }}(1)=1$, since $100 \%$ of elements from $S$ got a mark $\geq 10$, and

$\mu_{R \tilde{\div} S}(3)=\mu_{R \tilde{\div} S}(4)=\mu_{\text {Almost_all }}\left(\frac{4}{5}\right)=$ $\mu_{\text {Almost_all }}(0.8)=0.6$, since $80 \%$ of elements from $S$ got a mark $\geq 10$.

It can be checked that this tolerant division delivers elements (such as students \#3 and \#4) which

\begin{tabular}{|c|c|}
\hline \#Student & $\mu_{\text {StudentResults }} \geqslant 10$ \\
\hline \hline 1 & 1 \\
\hline 2 & 1 \\
\hline 3 & 0.6 \\
\hline 4 & 0.6 \\
\hline
\end{tabular}

Table 5: The resulting relation from a tolerant di-

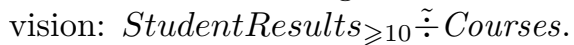

\begin{tabular}{|c|c|c|}
\hline \#Student & $\mu_{R_{\geqslant 10}} \tilde{\div} S$ & $\mu_{R_{\geqslant 15}} \tilde{\div} S$ \\
\hline \hline 2 & 1 & 0.6 \\
\hline 1 & 1 & 0.2 \\
\hline 3 & 0.6 & 0.6 \\
\hline 4 & 0.6 & 0.2 \\
\hline
\end{tabular}

Table 6: The resulting relation from: $\left(R_{\geq 10}, R_{\geq 15}\right) \stackrel{\sim}{\div}$ Courses.

would be discarded by the regular division.

A drawback of this tolerant division is that elements are ranked with respect to $\mu_{\text {Almost_all }}\left(\frac{v}{n}\right)$, which in fact reverts to rank the elements with respect to $\frac{v}{n}$ (as far as $\frac{v}{n} \neq 0$ ). We think that it is rather a weak discrimination and the next subsection shows that bipolarity can help to provide a refinement of this tolerant division.

\subsection{A tolerant bipolar relational division}

As pointed out, the tolerant division operator applied on crisp relations $R$ and $S$ delivers elements $x$ attached to degrees corresponding to portions of elements of $S$ which are related to $x$ in $R$. The obtained elements can be gathered with respect to the degrees, which leads to a weak discrimination between tuples. We show through the following example 3 that the bipolarity can improve the discrimination between the resulting tuples.

Example 3: The query of the example 2 can be extended to bipolarity as follows: "Find students who got a mark which is $\geq 10$ and if possible which is $\geq 15$ in almost all courses". The obtained query can be rewritten as "Find students who got a mark which is $\geq 10$ in almost all courses, and if possible students who got a mark which is $\geq 15$ in almost all courses".

The resulting relation is a fuzzy bipolar relation (see table 6) made of tuples from the tolerant division $R_{\geq 10} \tilde{\div} S$, and if possible tuples from the tolerant division $R_{\geq 15} \stackrel{\sim}{\div} S$, where $R_{\geq 10}$ (resp. $R_{>15}$ ) is the projection on columns \#Student and \# Course of the relation StudentResults restricted to students who got marks $\geq 10$ (resp. $\geq 15$ ), and $S$ denotes the relation Courses.

Each student is attached to two degrees, each of them being the expression of the tolerant division as previously defined ("find students who got a mark which is $\geq 10$ in almost all courses" and "find stu- 
dents who got a mark which is $\geq 15$ in almost all courses" respectively). The most important result is the one provided by the first division. The second degree may be used to discriminate between elements having the same level of satisfaction with respect to the first one. In other words, the lexicographical order can be used to rank the tuples and to provide a refinement to the order delivered by the tolerant division "find students who got a mark which is $\geq 10$ in almost all courses". Students are then ranked as follows: $\# 2>\# 1>\# 3>\# 4$.

More formally, this type of tolerant bipolar division can be defined as $\left(R_{1}, R_{2}\right) \stackrel{\tilde{\div}}{\div} S$ where $R_{1}$ and $R_{2}$ are two boolean relations such that $R_{2} \subseteq R_{1}$, and $S$ is a boolean relation. This kind of division expresses queries of the form "find elements from $R_{1}$ and if possible elements from $R_{2}$ which are related to almost all elements of $S$ ". This form is rewritten as: "find elements from $R_{1}$ which are related to almost all elements of $S$, and if possible elements from $R_{2}$ which are related to almost all elements of $S$ ". Such a query is interpreted as: for each delivered element $x$, "almost all elements of $S$ are associated with $x$ in $R_{1}$, and if possible almost all elements of $S$ are associated with $x$ in $R_{2}$ ".

This type of tolerant division can be rewritten $R_{(c, w)} \stackrel{\tilde{\div}}{\div} S$, where $R$ is a bipolar relation of schema $(A, X)$ defined by a boolean constraint $c$ and a boolean wish $w$, and $S$ is a boolean relation of schema $B$. In this case, the division of the bipolar relation $R_{(c, w)}$ by the boolean relation $S$ delivers elements $x$ from $R_{c}[X]$, which are related to almost all elements of $S$, and if possible, elements $x$ from $R_{w}[X]$, which are related to almost all elements of $S$. The resulting relation is a fuzzy bipolar relation and the pair of grades $\left(\mu_{R_{c} \tilde{\div} S}, \mu_{R_{w} \tilde{\div} S}\right)$ associated to tuples is computed as follows:

$$
\begin{aligned}
& \mu_{R_{c}[A \stackrel{\sim}{\div} B] S}(x)=\mu_{\text {Almost_all }}\left(\frac{v}{n}\right) . \\
& \mu_{R_{w}[A \tilde{\div} B] S}(x)=\mu_{\text {Almost_all }}\left(\frac{v^{\prime}}{n}\right),
\end{aligned}
$$

where $v$ (resp. $v^{\prime}$ ) is the number of elements of $S$ which are in relationship $R_{c}$ (resp. $R_{w}$ ) with $x$, and $n$ is the cardinality of $S$.

The obtained pairs of degrees satisfy the coherence property of fuzzy bipolar conditions, since $R_{w} \subseteq R_{c} \Rightarrow \forall x, v^{\prime} \leq v \Rightarrow \forall x$,Almost_all $\left(\frac{v^{\prime}}{n}\right) \leq$ Almost_all $\left(\frac{v}{n}\right)$.

It is worth noticing that the extension of such a tolerant bipolar division to fuzzy relations is rather difficult since its interpretation amounts to evaluate a quantified proposition of the form "Almost_all $Y X$ are $Z^{\prime \prime}$. This kind of quantified propositions raises an issue of interpretation when the fuzzy set $Y$ tends to an empty set. This case is not detailed because it is out of the scope of this paper.

\section{The division of fuzzy bipolar relations}

Different forms of divisions involving bipolar relations can be distinguished; they are studied in this section in terms of interpretation and evaluation.

\subsection{Division of the form $R \div S_{(c, w)}$}

By definition, the division operation is a matter of quantities. The division $R[A \div B] S$ delivers the $R[X]$ elements which are related in $R$ to all elements of $S$ without taking into consideration the meaning of this relationship. Therefore, in the bipolarity context, the division operator can be applied to express bipolar queries involving fuzzy relations $S_{1}$ and $S_{2}$ such that $S_{2} \subseteq S_{1}$ as divisors.

Such queries, which express a division, have then the following main format: "Return elements from $R[X]$ which are related to all elements of $S_{2}$ and if possible related to all elements of $S_{1}$ ".

More formally, let $R$ be a relation (either boolean or fuzzy) and let $S_{1}$ and $S_{2}$ such that $S_{2} \subseteq S_{1}$ be two fuzzy relations involved as divisors in a fuzzy bipolar division query of the form $R \div\left(S_{1}, S_{2}\right)$. This division is interpreted as follows:

$$
R \div\left(S_{1}, S_{2}\right)=\left(R \div S_{2}, R \div S_{1}\right)
$$

where $\left(R \div S_{2}, R \div S_{1}\right)$ denotes the fuzzy bipolar relation defined as elements which are from $R \div S_{2}$ and if possible elements which are from $R \div S_{1}$.

This type of division can be rewritten $R \div S_{(c, w)}$, where $R$ can be either a boolean or a fuzzy relation and $S$ is a fuzzy bipolar relation. In this case, the division of the relation $R(A, X)$ by the fuzzy bipolar relation $S_{(c, w)}$ delivers elements $x$ from $R[X]$, which are related to every element of $S_{w}$, and if possible, elements $x$ from $R[X]$, which are related to every element of $S_{c}$. The resulting relation is a bipolar relation and the pair of grades $\left(\mu_{R \div S_{w}}, \mu_{R \div S_{c}}\right)$ associated to tuples is computed as follows:

$$
\mu_{R \div S_{w}}(x)=\min _{a \in S_{w}}\left(\mu_{w}(a) \rightarrow \mu_{R}(a, x)\right),
$$$$
\mu_{R \div S_{c}}(x)=\min _{a \in S_{c}}\left(\mu_{c}(a) \rightarrow \mu_{R}(a, x)\right) \text {, }
$$

where $S_{c}$ (resp. $\left.S_{w}\right)$ is the projection of $S_{(c, w)}$ on the constraint $c$ (resp. on the wish $w$ ), and $\rightarrow$ is a fuzzy implication.

The obtained pairs of grades satisfy the coherence property of fuzzy bipolar conditions, since:

$$
\begin{aligned}
& \forall a \in S, \mu_{w}(a) \leq \mu_{c}(a) \text { then } \\
& \quad \forall x, \mu_{w}(a) \rightarrow \mu_{R}(a, x) \geq \mu_{c}(a) \rightarrow \mu_{R}(a, x) .
\end{aligned}
$$

Example 4: The following query "Find students who are well scored in all difficult courses and if possible in all very difficult courses" is interpreted as "Find students who are well scored in all very difficult courses, and if possible students who are well scored in all difficult courses" (all very difficult courses being included in all difficult courses). It corresponds to the bipolar division of the fuzzy relation StudentResults ${ }_{W e l l}$, denoted $R_{W e l l}$, by the fuzzy bipolar relation Courses (Difficult, VeryDifficult), denoted $S_{(\text {Dif, VeryDif) }}$ :

$$
\begin{aligned}
& R_{\text {Well }} \div S_{(\text {Dif }, \text { VeryDif })}= \\
& \quad\left(R_{\text {Well }} \div S_{\text {VeryDif }}, R_{\text {Well }} \div S_{\text {Dif }}\right) .
\end{aligned}
$$

Tables 7 and 8 display respectively an example of the relation Courses (Difficult,VeryDifficult) and the 


\begin{tabular}{|c|c|c|}
\hline \#Course & $\mu_{\text {Difficult }}$ & $\mu_{\text {VeryDifficult }}$ \\
\hline \hline$C_{1}$ & 1 & 1 \\
\hline$C_{2}$ & 0.5 & 0.25 \\
\hline$C_{3}$ & 0.8 & 0.64 \\
\hline
\end{tabular}

Table 7: Extension of the relation $S_{(D i f, \text { VeryDif) }}$.

\begin{tabular}{|c|c|c|}
\hline \#Student & $\mu_{R_{\text {Well }}} \div S_{\text {VeryDif }}$ & $\mu_{R_{\text {Well }}} \div S_{\text {Dif }}$ \\
\hline \hline 1 & 1 & 1 \\
\hline 2 & 0.9 & 0.9 \\
\hline
\end{tabular}

Table 8: The resulting relation from $R_{\text {Well }} \div$ $S_{(\text {Dif, VeryDif) }}$.

fuzzy bipolar relation which results from the division of $R_{\text {Well }}$ (see table 1) by the fuzzy bipolar relation $S_{(D i f, \text { VeryDif) }}$, when the Gödel fuzzy implication is used.

\subsection{Division of the form $R_{(c, w)} \div S$}

Let $R_{1}$ and $R_{2}$ be two fuzzy relations, such that $R_{2} \subseteq R_{1}$, involved as dividends in a fuzzy bipolar division of the form $\left(R_{1}, R_{2}\right) \div S$, where $S$ is either a boolean or a fuzzy relation, the division query delivers "elements of $R_{1}$ which are related to all elements of $S$ and if possible elements of $R_{2}$ which are related to all elements of $S$ ". More formally, this division is interpreted as follows:

$$
\left(R_{1}, R_{2}\right) \div S=\left(R_{1} \div S, R_{2} \div S\right),
$$

where $\left(R_{1} \div S, R_{2} \div S\right)$ is the fuzzy bipolar relation defined as elements which are from $R_{1} \div S$, and if possible elements which are from $R_{2} \div S$.

This type of division can be rewritten $R_{(c, w)} \div S$, where $R_{(c, w)}(A, X)$ is a fuzzy bipolar relation and $S$ is either a boolean or a fuzzy relation. In this case, the division of $R_{(c, w)}$ by the relation $S$ consists in the retrieval of elements $x$ of $R_{c}[X]$, which are related to all elements of $S$, and if possible, elements $x$ of $R_{w}[X]$, which are related to all elements of $S$.

The resulting relation is a fuzzy bipolar relation and the pair of grades $\left(\mu_{R_{c} \div S}, \mu_{R_{w}} \div S\right)$ associated to tuples is computed as follows:

$$
\begin{aligned}
& \mu_{R_{c} \div S}(x)=\min _{a \in S}\left(\mu_{S}(a) \rightarrow \mu_{c}(a, x)\right) . \\
& \mu_{R_{w} \div S}(x)=\min _{a \in S}\left(\mu_{S}(a) \rightarrow \mu_{w}(a, x)\right) .
\end{aligned}
$$

The obtained pairs of grades satisfy the coherence property of fuzzy bipolar conditions, since:

$\forall y \in R, \mu_{w}(y) \leq \mu_{c}(y)$ then

$\forall y=(a, x), \mu_{S}(a) \rightarrow \mu_{w}(a, x) \leq \mu_{S}(a) \rightarrow \mu_{c}(a, x)$.

Example 5: The following query "Find students who are well scored and if possible very well scored in all difficult courses" is rewritten as "Find students who are well scored in all difficult courses, and if possible students who are very well scored in all difficult courses". This query is processed as the division of the fuzzy bipolar relation StudentResults (WellScored, VeryWellScored), de-

\begin{tabular}{|c|c|c|c|}
\hline \#Student & \#Course & $\mu_{\text {WellScored }}$ & $\mu_{\text {Very WellScored }}$ \\
\hline \hline 1 & $C_{1}$ & 1 & 1 \\
\hline 1 & $C_{2}$ & 0.6 & 0.36 \\
\hline 1 & $C_{3}$ & 0.9 & 0.81 \\
\hline 2 & $C_{1}$ & 0.9 & 0.81 \\
\hline 2 & $C_{2}$ & 0.6 & 0.36 \\
\hline 2 & $C_{3}$ & 0.8 & 0.64 \\
\hline 3 & $C_{1}$ & 0.4 & 0.16 \\
\hline 3 & $C_{3}$ & 0.8 & 0.64 \\
\hline
\end{tabular}

Table 9: Extension of the relation $R_{(\text {Well, Very Well })}$.

\begin{tabular}{|c|c|c|}
\hline \#Student & $\mu_{R_{\text {Well }} \div S_{\text {Dif }}}$ & $\mu_{R_{\text {Very Well }} \div S_{\text {Dif }}}$ \\
\hline \hline 1 & 1 & 0.36 \\
\hline 2 & 0.9 & 0.36 \\
\hline
\end{tabular}

Table 10: The resulting relation from $R_{(\text {Well, Very Well })} \div S_{\text {Dif }}$

noted $R_{(\text {Well }, \text { VeryWell })}$ by the fuzzy relation Courses $_{\text {Difficult }}$, denoted $S_{\text {Dif }}$ (see table 2):

$R_{(\text {Well, Very Well })} \div S_{\text {Dif }}=$

$\left(R_{\text {Well }} \div S_{\text {Dif }}, R_{\text {Very Well }} \div S_{\text {Dif }}\right)$.

An example of extension of the relation $R_{(\text {Well, } \text { Very Well })}$ is displayed in table 9 . Table 10 shows the resulting relation when the Gödel fuzzy implication is used.

\subsection{Division of the form $R_{(c, w)} \div S_{\left(c^{\prime}, w^{\prime}\right)}$}

Let $R_{1}, R_{2}, S_{1}$ and $S_{2}$ be four fuzzy relations such that $R_{2} \subseteq R_{1}$ and $S_{2} \subseteq S_{1}$, which are involved in the bipolar division $\left(R_{1}, R_{2}\right) \div\left(S_{1}, S_{2}\right)$. Firstly, we consider the dividend $\left(R_{1}, R_{2}\right)$ and we apply the formula (7). Then, since $\left(S_{1}, S_{2}\right)$ is the divisor, the formula (6) is applied. Finally, the resulting fuzzy bipolar relation is obtained by identification.

Since $R_{1}$ and $R_{2}$ are involved in the bipolar division $\left(R_{1}, R_{2}\right) \div\left(S_{1}, S_{2}\right)$ as dividends and by using the formula (7), this fuzzy bipolar division must deliver a fuzzy bipolar relation $\rho$ defined by elements which are from $\left(R_{1} \div \Delta\right)$ and if possible elements from $\left(R_{2} \div \Delta^{\prime}\right): \rho=\left(R_{1} \div \Delta, R_{2} \div \Delta^{\prime}\right)$, where $\Delta$ and $\Delta^{\prime}$ are fuzzy relations.

Moreover, because $S_{1}$ and $S_{2}$ are involved as divisors and by using the formula (6), the bipolar division must deliver a bipolar relation $\rho^{\prime}$ defined by elements which are from $\left(\nabla \div S_{2}\right)$ and if possible elements from $\left(\nabla^{\prime} \div S_{1}\right): \rho^{\prime}=\left(\nabla \div S_{2}, \nabla^{\prime} \div S_{1}\right)$, where $\nabla$ and $\nabla^{\prime}$ are fuzzy relations.

Since, the fuzzy bipolar division $\left(R_{1}, R_{2}\right) \div$ $\left(S_{1}, S_{2}\right)$ delivers the same fuzzy bipolar relation as result, then $\rho=\rho^{\prime}$, which means that:

$\left(R_{1} \div \Delta, R_{2} \div \Delta^{\prime}\right)=\left(\nabla \div S_{2}, \nabla^{\prime} \div S_{1}\right)$.

By identification, we obtain straightforwardly:

$\Delta=S_{2}$ and $\Delta^{\prime}=S_{1}$

$\nabla=R_{1}$ and $\nabla^{\prime}=R_{2}$

Finally, the following general form for the division that involves fuzzy bipolar relations is obtained: 


\begin{tabular}{|c|c|c|}
\hline \#Student & $\mu_{R_{\text {Well }}} \div S_{\text {VeryDif }}$ & $\mu_{R_{\text {VeryWell }} \div S_{\text {Dif }}}$ \\
\hline \hline 1 & 1 & 0.36 \\
\hline 2 & 0.9 & 0.36 \\
\hline
\end{tabular}

Table 11: The resulting relation from $R_{(\text {Well, VeryWell })} \div S_{(\text {Dif, VeryDif })}$.

$$
\left(R_{1}, R_{2}\right) \div\left(S_{1}, S_{2}\right)=\left(R_{1} \div S_{2}, R_{2} \div S_{1}\right)
$$

This form of bipolar division can be rewritten $R_{(c, w)} \div S_{\left(c^{\prime}, w^{\prime}\right)}$, where both $R_{(c, w)}$ and $S_{\left(c^{\prime}, w^{\prime}\right)}$ are fuzzy bipolar relations. The division of $R_{(c, w)}$ of schema $(A, X)$ by the fuzzy bipolar relation $S_{\left(c^{\prime}, w^{\prime}\right)}$ delivers elements $x$ of $R_{c}[X]$ which are related to all elements of $S_{w^{\prime}}$, and if possible, elements $x$ of $R_{w}[X]$, which are related to all elements of $S_{c^{\prime}}$. The resulting relation is a fuzzy bipolar relation and the pair of grades $\left(\mu_{R_{c} \div S_{w^{\prime}}}, \mu_{R_{w} \div S_{c^{\prime}}}\right)$ associated to tuples is computed using formulas (1) and (2).

This form of fuzzy bipolar division is in adequacy with the general model introduced in $[4,5]$, and pairs of grades attached to answers satisfy the coherence property of fuzzy bipolar conditions as previously stated in subsection 3.1 .

Example 6: The following query "Find students who are (well scored and if possible very well scored) in all (difficult courses and if possible in all very difficult courses)" is rewritten as "Find students who are well scored in all very difficult courses, and if possible students who are very well scored in all difficult courses". This query is the division of the fuzzy bipolar relation StudentResults (WellScored, VeryWellScored), denoted $R_{(\text {Well }, \text { Very Well })}$, by the fuzzy bipolar relation Courses(Difficult, VeryDifficult), denoted $S_{(\text {Dif, VeryDif) }}$ :

$$
R_{(\text {Well, VeryWell })} \div S_{(\text {Dif }, \text { VeryDif })}=
$$$$
\left(R_{\text {Well }} \div S_{\text {VeryImp }}, R_{\text {VeryWell }} \div S_{\text {Dif }}\right) \text {. }
$$

Table 11 displays the fuzzy bipolar relation which results from the division of the fuzzy bipolar relation StudentResults (WellScored, VeryWellScored) (see table 9) by the fuzzy bipolar relation

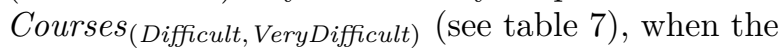
Gödel fuzzy implication is used.

\section{Conclusion}

The extension of the relational division operator to bipolarity is tackled in this paper. Firstly, a tolerant division operator and its extension to bipolarity are introduced. Secondly, a quantity-based division operator of fuzzy bipolar relations is studied in terms of interpretations. By doing this, we have shown that the general model proposed for the bipolar division $[4,5]$ can be interpreted in terms of bipolar statements, i.e. statements based on bipolar conditions of type "and if possible".

As future work, we aim at integrating of these forms of division operation into the bipolar SQLf language, which is an extension of the SQLf language $[3,2]$ to fuzzy bipolar conditions.

\section{Acknowledgments}

This work is funded by the Brittany region and the department of Côtes-d'Armor and is partially supported by the National Agency for Research (AOC Ref. ANR-08-CORD-009).

\section{References}

[1] G. Bordogna and G. Pasi. A fuzzy query language with a linguistic hierarchical aggregator In proceedings ACM SAC, 184-187, 1994.

[2] P. Bosc, L. Liétard, O. Pivert, and D. Rocacher. Base de données - Gradualité et imprécision dans les bases de données Ensembles flous, requêtes flexibles et interrogation de données mal connues. Technosup. 1 edition, 2004.

[3] P. Bosc and O. Pivert. SQLf: A relational database langage for fuzzy querying. IEEE Trans. on Fuzzy Systems, 3(1):1-17, Feb 1995.

[4] P. Bosc and O. Pivert. About bipolar division operators. FQAS 2009, LNAI 5822, 5822:572582, 2009.

[5] P. Bosc and O. Pivert. On the division of bipolar fuzzy relations. In proc. of NAFIPS, 2010.

[6] P. Bosc, O. Pivert, L. Liétard, and A. Mokhtari. Extending relational algebra to handle bipolarity. In 25th ACM $S A C^{\prime} 10$, pages 1717-1721, 2010.

[7] P. Bosc, O. Pivert, and D. Rocacher. About quotient and division of crisp and fuzzy relations. International Journal of Intelligent Systems, 29:185-210, 2007.

[8] D. Dubois and H. Prade. Bipolarity in flexible querying. LNAI, 2522:174-182, 2002.

[9] D. Dubois and H. Prade. Bipolarité dans un processus d'interrogation flexible. In porc. of LFA, 2002.

[10] L. Liétard and D. Rocacher. On the definition of extended norms and co-norms to aggregate fuzzy bipolar conditions. In proc. of IFSA/EUSFLAT, pages 513-518, 2009.

[11] L. Liétard, D. Rocacher, and P. Bosc. On the extension of SQL to fuzzy bipolar conditions. In proc. of the 28th NAFIPS'09, 2009.

[12] G. D. Tré, S. Zadrozny, T. Matthé, J. Kacprzyk, and A. Bronselaer. Dealing with positive and negative query criteria in fuzzy database quering bipolar satisfaction degrees. LNAI, FQAS, 5822:593-604, 2009.

[13] S. Zadrozny and J. Kacprzyk. Bipolar queries using various interpretations of logical connectives. LNAI, IFSA, 4529:182-190, 2007.

[14] S. Zadrozny and J. Kacprzyk. Bipolar queries: A way to enhance the flexibility of database queries. Advances in Data Management, SCI, 223:49-66, 2009. 